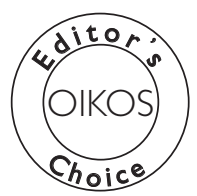

Oikos 123: 1409-1419, 2014

doi: 10.1111/oik.01767

(C) 2014 The Authors. Oikos (C) 2014 Nordic Society Oikos

Subject Editor: Timothy Cavagnaro. Editor-in-Chief: Dries Bonte. Accepted 10 July 2014

\title{
Most soil trophic guilds increase plant growth: a meta-analytical review
}

\author{
Andrew Kulmatiski, Andrew Anderson-Smith, Karen H. Beard, Stephen Doucette-Riise, \\ Michael Mazzacavallo, Nicole E. Nolan, Ricardo A. Ramirez and John R. Stevens
}

\begin{abstract}
A. Kulmatiski (andrewkulmatiski@hotmail.com), K. H. Beard and N. E. Nolan, Dept of Wildland Resources and the Ecology Center, Utah State Univ., Logan, UT 84322-5230, USA. - A. Anderson-Smith and M. Mazzacavallo, Dept of Biological Sciences, Univ. of Alaska Anchorage, Anchorage, AK 99508, USA. - S. Doucette-Riise, Plants, Soils and Climate Dept, Utah State Univ., Logan, UT 84322-4820, USA. - R. A. Ramirez, Dept of Biology Sciences, Utah State Univ., Logan, UT 84322-5305, USA. - J. R. Stevens, Dept of Mathematics and Statistics, Utah State Univ., Logan, UT 84322-3900, USA.
\end{abstract}

\begin{abstract}
Trophic cascades are important drivers of plant and animal abundances in aquatic and aboveground systems, but in soils trophic cascades have been thought to be of limited importance due to omnivory and other factors. Here we use a metaanalysis of 215 studies with 1526 experiments that measured plant growth responses to additions or removals of soil organisms to test how different soil trophic levels affect plant growth. Consistent with the trophic cascade hypothesis, we found that herbivores and plant pathogens (henceforth pests) decreased plant growth and that predators of pests increased plant growth. The magnitude of this trophic cascade was similar to that reported for aboveground systems. In contrast, we did not find evidence for trophic cascades in decomposer- and symbiont-based (henceforth mutualist) food chains. In these food chains, mutualists increased plant growth and predators of mutualists also increased plant growth, presumably by increasing nutrient cycling rates. Therefore, mutualists, predators of mutualists and predators of pests all increased plant growth. Further, experiments that added multiple organisms from different trophic levels also increased plant growth. As a result, across the dataset, soil organisms increased plant growth $29 \%$ and non-pest soil organisms increased plant growth $46 \%$. Omnivory has traditionally been thought to confound soil trophic dynamics, but here we suggest that omnivory allows for a simplified perspective of soil food webs - one in which most soil organisms increase plant growth by preying on pests or increasing nutrient cycling rates. An implication of this perspective is that processes that decrease soil organism abundance (e.g. soil tillage) are likely to decrease aboveground productivity.
\end{abstract}

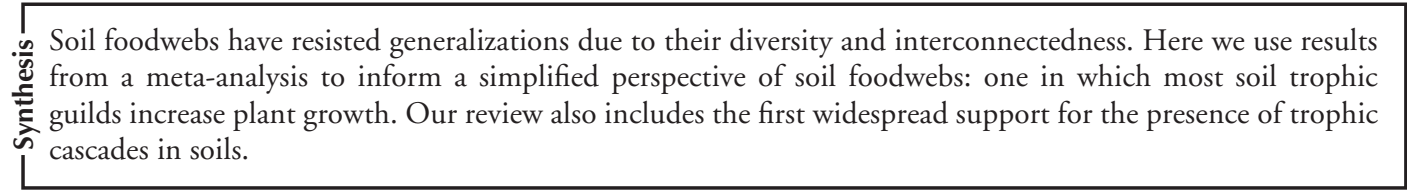

There is a growing appreciation for the fact that changing soil organism abundance and community composition can change plant productivity (Bever et al. 2010, Eisenhauer et al. 2012, Kulmatiski et al. 2012, Van der Putten et al. 2013). However, due to the difficulties inherent in working in dense, dark and diverse soils, most research on plant-soil interactions either treats soils as a 'black-box' (i.e. plantsoil feedback research) or focuses on the effects of specific soil pathogens or symbionts (e.g. plant disease research). Less is known about how ecological interactions in soils affect plant growth (Wardle 2006, Fierer et al. 2009, Strong and Frank 2010, Schneider et al. 2012). As a result, the consequences of intended (e.g. pesticide use) and unintended (e.g. climate) changes to soil communities are poorly understood (Lang et al. 2014).
In aquatic and aboveground terrestrial systems, trophic cascades help explain how biomass is distributed among plants and animals (Hairston et al. 1960, Paine 1980, Borer et al. 2005, Schmitz et al. 2006, Strickland et al. 2013). Trophic cascades are expected to be more important in systems with 1) homogeneous habitats, 2) fast prey population dynamics, 3) uniformly edible prey, 4) distinct trophic levels and 5) low predator and prey diversity (Polis 1999, Balvanera et al. 2006, Letourneau et al. 2009, Schneider et al. 2012). These conditions are more likely to be met in aquatic than terrestrial systems and consistent with this, trophic cascades are often stronger in aquatic systems (Polis 1999, Shurin et al. 2002).

High species diversity (Fierer et al. 2009), habitat heterogeneity (Ettema and Wardle 2002, Franklin and Mills 
2003), omnivory (Moore et al. 2004, Klarner et al. 2013) and variation in prey palatability (Klironomos and Kendrick 1996) are all likely to suppress trophic cascades in soils (Polis and Strong 1996, Hunt and Wall 2002). Further, compared to other systems, symbionts, decomposers and their predators are a large and important component of soil systems (Wall and Moore 1999). While predators of symbionts and decomposers could be expected to decrease plant growth (Knight et al. 2006), in soils these predators have been found to increase plant growth by increasing nutrient cycling rates (Ingham et al. 1985, Hedlund and Öhrn 2000, Moore et al. 2003, Lenoir et al. 2007, Nieminen 2008). These conditions and mechanisms along with the fact that several experiments and reviews have failed to find trophic cascades in soils have led soil ecologists to believe that trophic cascades are not important in these systems (Polis and Strong 1996, Mikola and Setälä 1998, Bradford et al. 2002, Sackett et al. 2010).

Despite all the factors that are likely to limit trophic cascades in soils, there is reason to expect that trophic cascades may be important (Wardle et al. 1995, Moore et al. 2004, Van der Putten et al. 2004, Schneider et al. 2012). Soil herbivores and pathogens can have large negative effects on plant growth (Blossey and Hunt-Joshi 2003, Morris et al. 2007) and parasites and predators can decrease the abundance of these plant pests (Rosenheim 1998, Preisser 2003, Toepfer et al. 2009, Singh et al. 2012). The fact that damaged plant roots release organic compounds that recruit the enemies of belowground herbivores (Van Tol et al. 2001) further supports the idea that predators may suppress soil herbivory. In agricultural settings, this mechanism has not gone unnoticed. Pathogens of root herbivores are gaining attention as biocontrol agents (Denno et al. 2008).

While many studies have examined trophic interactions among soil organisms (Clarholm 1985, Mikola and Setälä 1998, Hedlund and Öhrn 2000, Lenoir et al. 2007, Nieminen 2008, Schneider et al. 2012), evidence for cascading trophic effects on plant growth outside of species-specific examples are still lacking (Sackett et al. 2010). Here we use meta-analysis to explore the literature for general patterns of trophic dynamics in both decomposer-based and pest-based soil trophic pathways. Our goal was to use meta-analysis to address whether or not changes in soil organism abundance affect plant growth. Using a meta-analytical approach to address this goal has the strength of potentially identifying broad patterns of trophic dynamics but cannot be used to describe the idiosyncrasies or mechanisms of particular soil food webs (Lenoir et al. 2007, Schneider et al. 2012). We largely followed the approach used by Sackett et al. (2010), who performed a meta-analytical review for trophic cascades focused on the decomposer food web. We built upon that review to explore the effects of a wider range of soil organisms on plant growth (e.g. herbivores, plant pathogens, mycorrhizae and earthworms).

\section{Material and methods}

We searched for manuscripts where soil organism abundance was increased or decreased and plant growth responses to treatment were measured. Treatments were typically of an inoculation type in which soil organisms were added to soils or of a removal type in which soil organisms were selectively removed using chemical (e.g. insecticides or fungicides) or physical (e.g. sieving or freezing) techniques.

All manuscripts were located by searching in ISI Web of Knowledge for the following keywords: Plant and Soil and each of the following individually: Aphid, Collembolan, Decomposer, Entomopathogenic, Earthworm, Enchytraeid, Fungicide, Herbivor*, Inoculation, Mesofauna, Microarthropod, Mite, Nematode, Oligochaeta, Predaceous, Predator, Predatory nematode, Protozoa, Rhizosphere, Root feeder, Root herbivore, Springtail, Steriliz* and 'Trophic cascade'. Results were sorted by topic to include: Environmental Sciences Ecology, Plant Sciences, Forestry, and Biodiversity Conservation. We excluded studies categorized as Agriculture and studies examining bioremediation because there is a strong potential for these systems to behave differently than more diverse and heterogeneous wildland systems. Searches were performed in March 2011 and August 2013.

We placed species into trophic categories using authordefined trophic descriptions because we felt that authors were in the best position to describe the role of species correctly within their study system. Using these descriptions, species were placed into the following broad trophic categories: Symbionts, Decomposers, Predators of Mutualists (predators of Decomposers and Symbionts), Herbivores, Pathogens, Predators of Pests (predators of Herbivores and Pathogens) and Secondary Predators (described in more detail below). Earthworms were treated separately because they function as ecosystem engineers and generalist grazers and are difficult to categorize (Lavelle et al. 1997). Similarly, treatments that sterilized soils (Sterilized) and treatments that manipulated three or more species from two or more trophic categories (Community) were treated as separate categories. These latter two categories were expected to provide potentially important insight into soil organism effects but did not fit within the previously mentioned trophic categories. This long-used, simplified 'trophic guild' approach may be an oversimplification (Polis and Strong 1996), but is re-gaining attention as a tractable way to describe soil systems with complex food webs (Wollrab et al. 2012). Data were additionally categorized by ecosystem type (e.g. Forest, Grassland), plant type (e.g. Forb, Grass, Tree) and common organism type (e.g. Collembolan, Earthworm, Enchytraeid) using author descriptions.

To provide a clearer description of how soil organisms were classified into different trophic categories, here we present the sample sizes of the taxonomic groups that were placed into each trophic category. Each guild was typically dominated by one type of organism. Note that many experiments added more than one member of a guild so that sample sizes do not necessarily match the sample sizes reported in the results. The Herbivore group largely reflected nematode herbivores $(n=101)$, but also included beetles $(n=31)$, Collembolans $(n=18)$, Dipterans $(n=12)$, Enchytraeids $(n=3)$, moths $(n=4)$, hemiparasitic plants $(n=4)$, and mites $(n=2)$. Pathogens were primarily fungal $(n=41)$ but also included nematodes $(n=3)$. Predators of Pests were primarily entomopathogenic pathogens of herbivorous insects $(\mathrm{n}=34)$ but also included nematodes $(\mathrm{n}=11)$, beetles $(n=9)$, crickets $(n=6)$, bacteria $(n=5)$ and mites $(n=4)$. Predators of Mutualists were mostly Collembolans $(\mathrm{n}=37)$ but 
also included protozoa $(\mathrm{n}=15)$, nematodes $(\mathrm{n}=14)$ and beetles $(n=6)$. Decomposers were primarily Enchytraeids $(n=43)$ but also Collembolans $(n=38)$, bacteria $(n=24)$, fungi $(n=6)$, and mites $(n=6)$. The Symbiont group was primarily mycorrhizal fungi $(n=559)$ but also included endophyte fungi $(\mathrm{n}=3)$, gibberellin-producing, growth-promoting fungi (e.g. Phoma sp. GS8-2; $\mathrm{n}=6)$ and rhizobium $(\mathrm{n}=8)$.

\section{Data collection}

For each experiment, sample size $(n)$, mean $(\bar{X})$ and standard deviation $(S D)$ were recorded for control and experimental groups. 'Control' and 'experimental' groups are required by meta-analysis. In studies where organisms were removed, we arbitrarily assigned data from treatments that removed organisms to the 'control' group and data from treatments that did not remove soil organisms to the 'experimental' group. Similarly, in studies where organisms were added, we assigned data from treatments that did not add organisms to the 'control' group and data from treatments that added organisms to the 'experimental' group. In this way 'control' data always reflected the effects of fewer soil organisms on plant growth than 'experimental' data. While few studies assessed whether or not treatments effectively changed soil organism abundance, composition or diversity, we assume that 'experimental' treatments were effective (i.e. that treatments increased target organism abundance).

We treated experiments where investigators subjected different species to the same treatments, or the same species to different treatments as separate experiments (Gurevitch and Hedges 1999, Gurevitch et al. 2001). Data from these studies, however, were down-weighted using a Bayesian model to account for within-study biases (Stevens and Taylor 2009). The response variable that linked best to aboveground plant biomass was used. Different response measures for the same experiment were not included, except when above- and belowground measures were collected from the same experiment because these were never analyzed together. We did not include studies that only measured plant damage because it is not clear if these effects translate to differences in plant biomass (Shurin et al. 2002). If response variables were measured more than once, the last response measured was used. Datathief III (Tummers 2006) software was used to extract values from figures. When necessary, data was requested from authors.

\section{Statistical analyses}

First, a mixed-model approach was performed using MetaWin ver. 2.1 Software (Rosenberg et al. 2000). This approach is widely used and allows comparison among different meta-analyses, but does not control for sampling or hierarchical dependence. To control for these effects (see below) we conducted a more conservative Bayesian modeling approach (Stevens and Taylor 2009).

In the mixed-model approach, the natural log value of the response ratio (LRR) was calculated for each experiment (Rosenberg et al. 2000) where $L R R=\ln \left(\bar{X}_{E}\right)-\ln \left(\bar{X}_{C}\right)$, and ln is the natural $\log$ and $\bar{X}_{E}$ and $\bar{X}_{C}$ are the mean response in the experimental and control treatments, respectively. Use of the LRR provides robust statistical properties and allows direct comparison with related meta-analyses (Schmitz et al. 2000, Shurin et al. 2002, Sackett et al. 2010). Positive treatment responses indicated that adding soil organisms increased plant growth, while negative treatment responses indicated that adding soil organisms decreased plant growth. To account for the precision of each study, the effect size variance $(v)$ was calculated as $v=\frac{S D_{E}^{2}}{n_{E} \bar{X}_{E}^{2}}+\frac{S D_{C}^{2}}{n_{C} \bar{X}_{C}^{2}}$ (Hedges and Olkin 1985), where $S D_{E}$ and $S D_{C}$ are the standard deviations, $n_{E}$ and $n_{C}$ are the sample sizes, respectively. This calculation weights experiments with higher replication and lower variance more heavily.

To account for the fact that effect sizes were likely to differ among studies due to differences in the species used or experimental design, we used a mixed-effect model to calculate effect sizes (Gurevitch and Hedges 1999). Mixedeffects models account for this variation by calculating between-study variance and weighting each experiment by the inverse sum of the individual experiment variance and the between experiment variance (Hedges and Olkin 1985). The $Q_{t}$ (total) statistic was calculated first to determine if there was heterogeneity in the mean effect size (Rosenberg et al. 2000). We assumed that significant heterogeneity could be explained by variation among grouping variables (e.g. trophic categories). Grouping variables were selected a priori as described above.

To test for differences among grouping variables $Q_{b}$ (between) and $Q_{e}$ (residual) were calculated. These values are similar to experimental and residual sums of squares used in ANOVA analyses. $Q_{b}$ statistics and associated $\mathrm{p}$-values were used to test for differences among grouping variables and whether within-group heterogeneity was significant using a $\chi^{2}$ test (Rosenberg et al. 2000). Mean total effect size (E++) and grouping-variable specific effect sizes $(\mathrm{E}+)$ and $95 \%$ confidence intervals are reported. To help deal with potential issues associated with non-normal data, confidence intervals were calculated using bias-corrected bootstrap resampling procedures with 999 iterations from the effect sizes and their variances (Adams et al. 1997). Confidence intervals that did not overlap zero were considered significant.

We also use a Bayesian modelling approach to perform meta-analyses that control for sampling and hierarchical dependences (Kulmatiski et al. 2008). It is common for studies to use the same sample as a control for more than one experimental group resulting in sampling dependence among the effect size estimates. Studies with this type of sampling dependence are sometimes referred to as "multiple-treatment studies" (Gleser and Olkin 1994). To account for sampling dependence, the estimated sampling covariance matrix $V$ of the effect size estimates was used. It can be shown that the variance of experiment i's effect size estimate $L R R_{i}$ can be estimated as $V_{i, i}=\frac{S D_{E}^{2}}{n_{E} \bar{X}_{E}^{2}}+\frac{S D_{C}^{2}}{n_{C} \bar{X}_{C}^{2}}$. When experiments $i$ and $h(i \neq h)$ have sampling dependence as described here (i.e. $L R R_{i}=\ln \left(\bar{X}_{i}\right)-\ln \left(\bar{X}_{C}\right)$ and $L R R_{b}=$ $\ln \left(\bar{X}_{b}\right)-\ln \left(\bar{X}_{C}\right)$ for two experimental sample groups $(i$ and $h)$ compared to the same control sample group $(C))$, it can also be shown that the covariance of the effect size estimates $L R R_{i}$ and $L R R_{h}$ can be estimated as $V_{i, h}=\frac{S D_{C}^{2}}{n_{C} \bar{X}_{C}^{2}}$. Alternative estimates 
of the variance/covariance structure such as those in Gleser and Olkin (1994) are similar to those used here. With this sampling covariance matrix the effect size estimates $L R R_{i}$ can be combined using a linear model.

Groups of experiments can be considered hierarchically dependent if they were performed as a batch of experiments by the same research team. For example, the Chen et al. (2006) study reports 61 experiments on eight fungal species and three different tree species with several different inoculation densities and spore storage conditions. Because these data came from the same research team at one time period, they can be considered as having come from the same batch of experiments and hence present the potential for hierarchical dependence. To address this dependence, we combine the effect size estimates from the multiple experiments using a hierarchical Bayes linear model (DuMouchel and Harris 1983, DuMouchel and Normand 2000). This model accounts for both sampling and hierarchical dependence (Stevens and Taylor 2009). This approach can be summarized in matrix form as the linear model $L R R=X \beta+\delta+\varepsilon$ where $d$ is the vector of effect size estimates (the LRR) from all the experiments, $X$ is a design matrix (to account for covariate effects), $\beta$ is a vector of parameters (the effects of the covariates), $\delta$ is a vector of hierarchical errors, and $\varepsilon$ is a vector of sampling errors. This model assumes the distributions $\begin{aligned} & \delta \sim N(0, \Delta(\tau, \varsigma)) \\ & \varepsilon \sim N(0, V)\end{aligned}$ where $V$ is the sampling covariance matrix defined above (with $v=\frac{S D_{E}^{2}}{n_{E} \bar{X}_{E}^{2}}+\frac{S D_{C}^{2}}{n_{C} \bar{X}_{C}^{2}}$ on the diagonal) and $\Delta$ is the hierarchical covariance matrix. Briefly, $\Delta$ is a block-diagonal matrix with hierarchical variance $\tau^{2}$ on the diagonal for all experiments and hierarchical covariance $\varsigma$ on the off-diagonal for pairs of experiments that are hierarchically dependent. The block-diagonal structure of $\Delta$ effectively splits the hierarchical errors $\delta_{i}$ into two components, a study-specific component and an experimentwithin-study component. For this reason, this approach may be referred to as "delta-splitting" (Stevens and Taylor 2009). The hierarchical dependence concerns the correlation among the experiment-within-study components. Stevens and Taylor (2009) provide additional details and interpretation, and this Bayes model with hierarchical dependence is implemented in the metahdep package (Stevens and Nicholas 2009) for R.

For each of our research questions, we defined a design matrix $X$ to include columns of 0 's and 1's representing indicator variables for specific covariate levels. For example, we used columns for Symbiont, Herbivore, Predator and Community trophic categories. Then each term in $\beta$ can be interpreted as the population mean effect size of the corresponding covariate level. Bayesian methods were used to make inference on the $\beta$, with a normal prior on $\beta \mid(\tau, \varsigma)$, a uniform prior on $\varsigma \mid \tau$, and a $\log$-logistic prior on $\tau$. This approach provided the posterior mean and covariance of $\beta$, along with the posterior probabilities for each component of $\beta$. To facilitate interpretation and comparison between models, the posterior probabilities were converted to two-sided p-values, as in (Louis and Zelterman 1994): $P_{j}=1-2 \mid 0.5$ $-\operatorname{pr}\left(\beta_{j}>0 \mid\right.$ data $) \mid$. These are reported in the Results section. Computational details of this approach are provided in Stevens and Taylor (2009). For visualization purposes, the square root of the posterior variance of each term in $\beta$ was also calculated and used as a standard error of the covariate levels' mean effect sizes. Results in the delta-splitting model were considered significant if $\mathrm{p}<0.10$ because of the conservative nature of the model.

Publication bias was tested using funnel plots, failsafe numbers and by removing experiments with large effect sizes (Rosenberg et al. 2000, Bancroft et al. 2007). A linear regression of funnel plot asymmetry (testing the intercept term in a Galbraith plot) was used to test if studies with small effect sizes or high variance were missing from the dataset (Egger et al. 1997). Fail-safe numbers are the number of non-significant studies that could be added to a metaanalysis dataset before results become non-significant (Rosenberg 2005). Finally, to test the robustness of our results, we 1) ranked data by the magnitude of effect sizes in each grouping variable, 2) removed the largest effect sizes, and 3) re-ran meta-analyses until confidence intervals overlapped zero (Bancroft et al. 2007). This helped ascertain if a few experiments with large effect sizes determine the results.

Our search produced 7582 manuscripts. Of these, 215 met our criteria and produced 1526 lines of data (i.e. experiments). Aboveground measurements $(\mathrm{n}=1193)$ were more common than belowground $(\mathrm{n}=239)$ or total biomass $(\mathrm{n}=94)$ measurements. Aboveground biomass measurements $(n=1333)$ were most common, with measures of seed mass $(n=42)$, plant height $(n=40)$, seed number $(n=40)$ and seed survival $(\mathrm{n}=32)$ less common. Other measures, such as flower mass or count, were also recorded but made up less than 3\% of the dataset. See Supplementary material Appendix 1 for the complete list of manuscripts used.

\section{Results}

Across the dataset plant growth was on average 29\% greater in experimental than control treatments (i.e.In line). In non-pest experiments, plant growth was $46 \%$ greater in experimental than control treatments. Using the mixedmodel, plant growth was greater in experimental than control treatments and there was significant heterogeneity in the dataset $\left(\mathrm{E}++=0.23, \mathrm{CI}=0.20\right.$ to $0.26 ; Q_{t}=1798$, $\mathrm{DF}=1270, \mathrm{p}<0.001)$. Aboveground growth responses and total growth responses were not different $\left(Q_{b}=2.48\right.$, $\mathrm{DF}=1, \mathrm{p}=0.27$ ) so these responses were combined for subsequent analyses.

In the mixed model, trophic categories differed in their effect on plant growth $\left(Q_{b}=212, \mathrm{DF}=7, \mathrm{p}=0.001\right)$. Symbionts, Decomposers, Earthworms, Predators of Mutualists (i.e. predators of Symbionts and Decomposers), Predators of Pests (i.e. predators of Herbivores and Pathogens) and Communities all increased plant growth, and Herbivores and Pathogens decreased plant growth (Fig. 1). While the conservative delta-splitting model tends to produce smaller effect size estimates, the general pattern of results from the delta-splitting model was consistent with results from the mixed model (Fig. 1). For each category the mixed model and the delta-splitting model had effect sizes that were significantly different and in the same direction ( $p<0.001$ for each category in the delta-splitting model). Only five experiments examined the effects of Secondary 


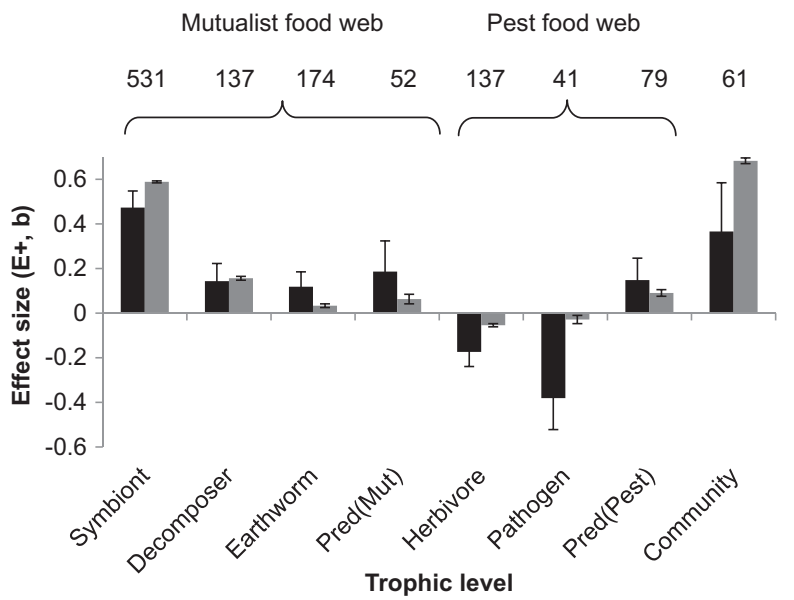

Figure 1. Overall effects of soil organism categories on aboveground plant growth determined from mixed-model (black bars) and deltasplitting (grey bars) meta-analytical models. The delta-splitting model uses a more conservative Bayesian model that accounts for sampling and hierarchical dependence. The Pred(Mut) category includes the effect of the addition of predators of Symbionts and predators of Decomposers. The Pred(Pest) category includes the effect of the addition of predators of Herbivores and predators of Pathogens. Error bars represent bias-bootstrapped 95\% confidence intervals. Data from trophic guilds in the 'Mutualist' pathway were combined to simplify analyses in subsequent analyses (i.e. to test for soil organism effects in different ecosystems and plant types). Data from the Herbivore and Pathogen categories in the 'Pest' pathway were similarly combined. The numbers above each bar indicate the number of experiments used to calculate each mean.

predators so these results were considered unreliable and were not presented.

To provide a simpler test of soil trophic effects in different ecosystems and on different plant types, we produced a simplified food web that combined Symbionts, Decomposers, Earthworms and Predators of Mutualists in a 'Mutualist' category. Similarly, data from Herbivores and Pathogens were included in a new 'Pest' category and data from Predators of Pest and Communities remained in their respective categories. This allowed us to compare four rather than eight 'trophic categories' (i.e. Mutualists, Pests, Predators of Pests and Communities).

Forests and Grasslands comprised 94\% of the data so other study systems (e.g. dunes) were not included in this analysis due to a lack of sample size. Overall, soil organisms had a more positive effect on plant growth in Forests $(\mathrm{E}+=0.29, \mathrm{CI}=0.24$ to 0.35$)$ than Grasslands $(\mathrm{E}+=0.18$, $\mathrm{CI}=0.12$ to $\left.0.24 ; Q_{b}=14, \mathrm{DF}=1, \mathrm{p}=0.009\right)$. In both Forests and Grasslands, Mutualists increased plant growth and Pests decreased plant growth (Fig. 2; $Q_{b}=191, \mathrm{DF}=9$, $\mathrm{p}=0.001$ ). Predators of Pests only had a significantly positive effect on plant growth in Forests, but consistent with the presence of trophic cascades, plant growth was greater in the Predators of Pests category than in the Pests category in both Grasslands and Forests (Fig. 2). Similarly, Community treatments increased plant growth in Forests but not Grasslands. Results from the delta-splitting model supported mixed model results for Forests (Supplementary material Appendix 2 Fig. A1). In Grasslands, however, the delta-splitting

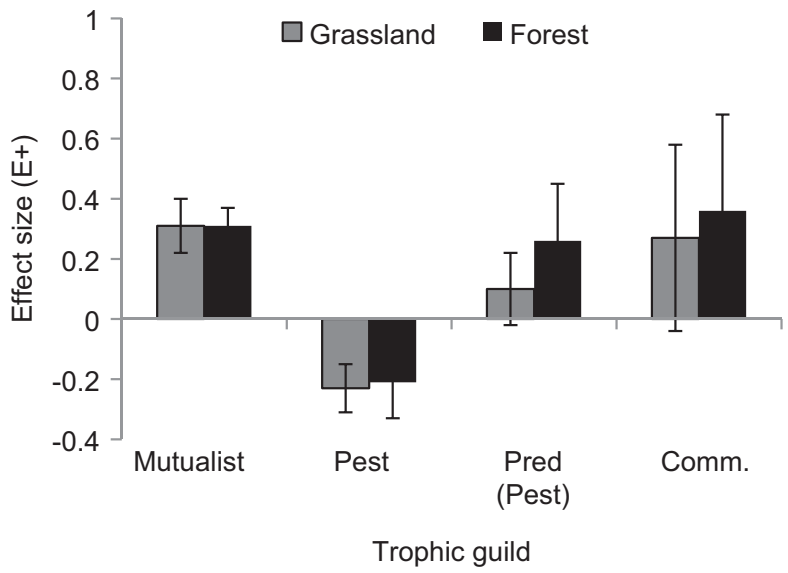

Figure 2. Effects of a simplified trophic guild structure on plant growth in grassland and forest ecosystems. The 'Mutualist' trophic guild represents the effects of mycorrhizae, saprobes, earthworms and saprobe predators as all these organism had positive effects on plant growth. The 'Pest' trophic guild represents the effects of both herbivores and pathogens on plant growth as these trophic guilds had similar negative effects on plant growth. The effects of predators of pests [Pred(Pest)] represents the effects of adding organisms that consume herbivores and plant pathogens. The effects of adding organisms from at least three different trophic guilds is shown in the Community column. Means and bias bootstrapped 95\% confidence intervals reported. The delta-splitting model produced similar results (Supplementary material Appendix 2).

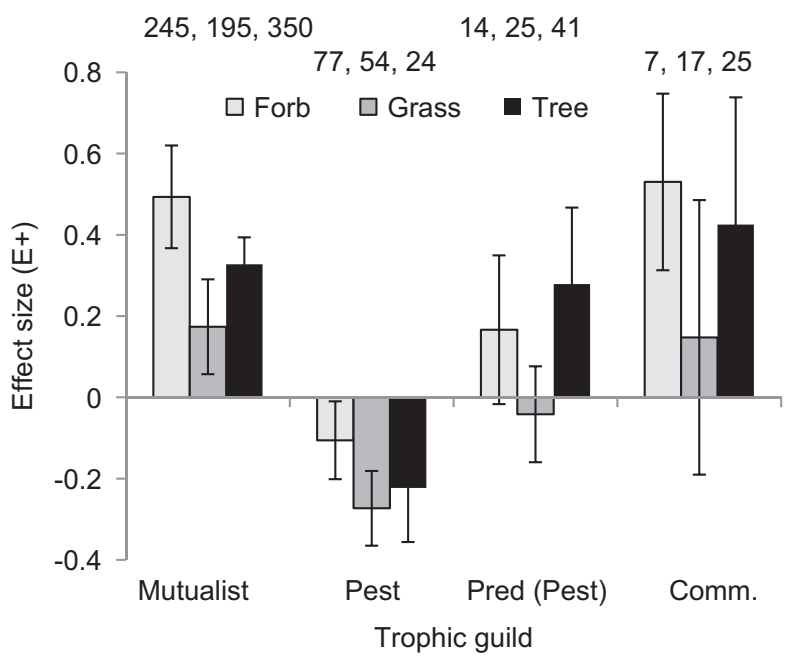

Figure 3. Effects of a simplified trophic guild structure on forb, grass and tree growth. The 'Mutualist' trophic guild represents the effects of mycorrhizae, saprobes, earthworms and saprobe predators as all these organism had positive effects on plant growth. The 'Pest' trophic guild represents the effects of both herbivores and pathogens on plant growth as these trophic guilds had similar negative effects on plant growth. The effects of predators of pests [Pred(Pest)] represents the effects of adding organisms that consume herbivores and plant pathogens. The effects of adding organisms from at least three different trophic guilds is shown in the Comm. column. Means and bias bootstrapped 95\% confidence intervals reported. The delta-splitting model produced similar results (Supplementary material Appendix 2).Values above each bar indicate the sample size associated with each grouping. 
model suggested that the presences of trophic cascades was due in part to sampling and hierarchical dependence. This suggests that more studies are needed to test the presence of trophic cascades in Grasslands suggested by the mixed model (Supplementary material Appendix 2 Fig. A2). The deltasplitting model also indicated that sampling and hierarchical dependence masked the positive effects of Communities on plant growth (i.e. Community effects were more positive than suggested by the mixed model).

When soil organism effects were tested on three plant life forms, Mutualists increased growth and Pests decreased growth of Forbs, Grasses and Trees (Fig. 3; $Q_{t}=2146$, $\left.\mathrm{DF}=1175, \mathrm{p}<0.001 ; Q_{b}=253, \mathrm{DF}=15, \mathrm{p}=0.001\right)$. For Grasses and Trees, plant growth was greater in Predators of Pests than Pests categories (Fig. 3). Predators of Pests increased growth of Trees. Communities increased growth of Forbs and Trees (Fig. 3). Again, the delta-splitting model supported mixed-model results and demonstrated greater growth of Forbs, Grasses and Trees in Predator of Pest than Pest treatments (Supplementary material Appendix 2 Fig. A2).

There was significant heterogeneity among the soil sterilization experiments, but the net effect was not different than zero $\left(\mathrm{E}++=0.08, \mathrm{CI}=-0.03\right.$ to $0.18 ; Q_{t}=224$, $\mathrm{DF}=81, \mathrm{p}<0.001)$. This reflected the fact that Trees realized a negative growth response to soil sterilization $(\mathrm{CI}=0.22$ to 0.37$)$ and Grasses realized a positive growth response to sterilization $\left(\mathrm{CI}=-0.39\right.$ to $-0.19 ; Q_{b}=200$, $\mathrm{DF}=1, \mathrm{p}=0.001$.

We also analyzed the effects of soil organism identity. Soil organisms differed in their effect on plant growth (Fig. 4; $\left.Q_{b}=142, \mathrm{DF}=6, \mathrm{p}=0.001\right)$. Arbuscular mycorrhizal fungi (AMF; $n=540$ ), Collembolans $(n=50)$, and earthworms $(n=191)$ had positive effects on plant growth. Non-mycorrhizal fungi $(n=71)$ had negative effects on plant growth. Enchytraeids $(n=42)$ and nematodes $(n=101)$ had no effect on plant growth (Fig. 4). Results from the deltasplitting model were consistent with the mixed model for AMF and Collembola but not for the other groups (Fig. 4). In contrast to the mixed-model, the delta-splitting model

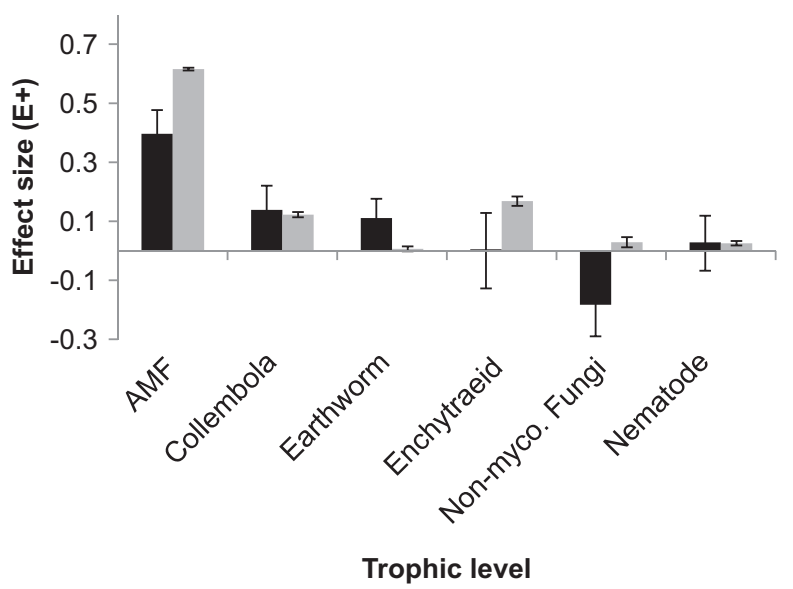

Figure 4. Effects of different soil organism taxonomic groups on plant growth determined using mixed-model (black bars) and delta-splitting (grey bars) meta-analytical models. Means and bias-bootstrapped $95 \%$ confidence intervals shown. indicated that Earthworms had no net effect on plant growth, Enchytraeids had a positive effect on plant growth, fungi had no effect on plant growth and nematodes had a positive effect on plant growth. The delta-splitting model controls for sampling and hierarchical dependence. Thus the difference between model results reflects the fact that some studies used the same control sample for multiple treatments (sampling dependence) and that a large number of experiments from one author skewed results. It is important to note that this hierarchical bias controlled by the delta-splitting model is different than 'file-drawer' bias (i.e. authors not publishing insignificant results) described below.

Across the dataset, greater soil organism abundance increased belowground plant growth but there was no significant heterogeneity in the dataset to suggest that different grouping variables had different effects on root growth $\left(\mathrm{E}++=0.19, \mathrm{CI}=0.11\right.$ to $0.28, Q_{t}=194$, $\mathrm{DF}=199, \mathrm{p}=0.587)$.

'File-drawer' bias did not appear important because studies with the smallest sample sizes or largest standard errors tended to have effect size estimates near zero whereas a publication bias would likely show larger (or smaller) effect size estimates with larger sample sizes or smaller standard errors. This conclusion was supported with analysis of a funnel plot and histogram that indicated a nearly normal distribution of effect sizes (Supplementary material Appendix 2 Fig. A3). A linear regression test of funnel plot asymmetry (testing the intercept term in a Galbraith plot) yielded a non-significant result $(\mathrm{p}=0.53$; Supplementary material Appendix 2 Fig. A3; Egger et al. 1997). Finally, Rosenthal's fail-safe numbers indicated that 64405 null studies would have to be added to the dataset to change the results. This suggested that results were robust to 'file-drawer' bias (Rosenthal 1979).

Results were not driven by large effect sizes. The confidence intervals for the Community, Earthworm, Herbivore, Pathogen, Predator of Pest, Decomposer, Predator of Mutualist and Symbiont effects would have remained different from zero even after removing 58, 83, 54, 73, 67, 23, 6 and 59\% of the largest effect sizes, respectively. Thus, only the Predator of Mutualist category was at all sensitive to a few large effect sizes, yet even half of the dataset with the smallest effect sizes showed a pattern of positive effects on plant growth $(\mathrm{E}+=0.03, \mathrm{CI}=-0.03$ to 0.10$)$. Thus, results were robust against 'file-drawer' bias and were unlikely to be driven by results from a few experiments with large effect sizes.

\section{Discussion}

\section{The cascading 'Pest' pathway and the 'Mutualist' pathway}

By separating the Pest and Mutualist food webs in this review (Fig. 5), we were able to investigate and find interactions unique to each food web. In the Pest food web, we found strong evidence for soil trophic cascades: 1) Herbivores and Pathogens (i.e. Pests) decreased plant growth, and 2) Predators of Pests increased plant growth. The size of this effect was similar to that observed in aboveground systems (i.e. the 

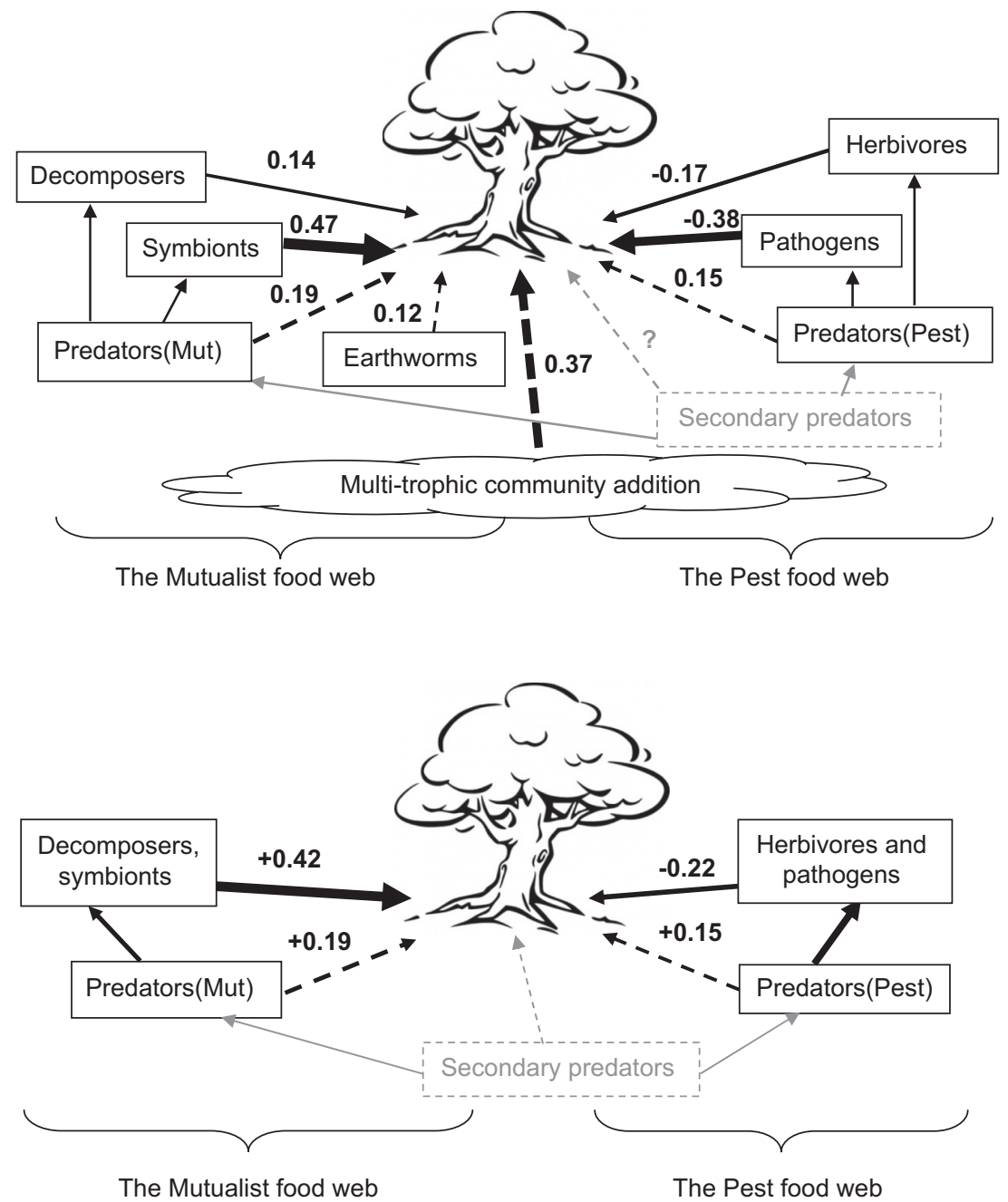

Figure 5. A full (a) and simplified (b) conceptual scheme of the soil trophic web examined in this meta-analysis. Experiments identified in the literature where categorized in one of the listed boxes and our meta-analysis was used to estimate the strength of these effects on plant growth. Solid lines indicate hypothesized direct effects and dashed lines indicate hypothesized indirect effects. Many other pathways are known to occur between compartments, but here we show those pathways that are hypothesized to be most likely to drive results observed in our meta-analysis. Secondary predators are shown in a grey hatched box because these organisms are expected to play a role in soil trophic dynamics but insufficient data were available in the literature to assess the roles of these organisms.

LRR was 0.15 in this study and 0.10 and 0.22 in reviews of aboveground predator effects; Schmitz et al. 2000, Shurin et al. 2002, respectively). These trophic cascade effects were stronger for Forbs and Trees than for Grasses. In contrast to the Pest food web, but consistent with previous studies (Mikola and Setälä 1998, Bradford et al. 2002, Hunt and Wall 2002, Sackett et al. 2010), we did not find evidence of soil trophic cascades in the Mutualist food web.

While trophic cascades were only apparent in the Pest food web, Predators in both the Pest and Mutualist food webs increased plant growth. This effect likely occurred through two different primary mechanisms. In the Pest food web, it is likely that plant growth increased through a classic trophic cascade. This has been shown in single studies (Preisser 2003, Singh et al. 2012), and with biocontrol agents (Denno et al. 2008, Toepfer et al. 2009), but we believe this is the first review showing soil trophic cascades in nonagricultural systems. Predators in the Mutualist food web also increased plant growth despite the fact that these preda- tors likely decrease Mutualist abundance. There is extensive support for the idea that Predators of Mutualists can increase plant growth by increasing nutrient cycling rates (Clarholm 1985, Mikola and Setälä 1998, Hedlund and Öhrn 2000, Lenoir et al. 2007, Nieminen 2008). This 'predator-mediated nutrient enrichment' has also been observed in aboveground systems and it may even explain a large portion of effects traditionally associated with trophic cascades (Sin et al. 2008).

Omnivory, prey defenses, diversity and habitat heterogeneity have all been suggested to preclude the development of trophic cascades in soils yet we observed surprisingly strong trophic cascade effects. We suggest three reasons trophic cascades were found in this review. First, we believe this is the first meta-analysis to separate the Pest and Mutualist pathways and this was critical to identifying trophic cascades in the Pest pathway (Sackett et al. 2010). Second, it is possible that belowground Pests have greater effects on plant growth than aboveground Pests (Jarosz and Davelos 1995, 
Klironomos 2002, Blossey and Hunt-Joshi 2003) so even if soil trophic cascades are weakened by omnivory, for example, the net effect of soil trophic cascades may be similar to the net effect of aboveground trophic cascades. Finally, predatormediated nutrient enrichment could be expected to be more important in soils than aboveground systems because soil predators release nutrients where they can be immediately absorbed by plant roots.

Additional mechanisms may also explain positive effects of soil predators on plants. Fungal foraging may select for mycorrhizae that induce greater benefits to plant hosts (Klironomos and Kendrick 1996, Klironomos 2003), large soil organisms may increase aeration and decomposition through disturbances and communition (Verhoef and Brussaard 1990) or other more idiosyncratic mechanisms may be important (Schneider et al. 2012, Zhao et al. 2013). Whether predators have positive effects on plant growth by suppressing herbivory, increasing nutrient cycling, stabilizing community interactions or some other mechanism cannot be determined from this analysis. However, our review shows that predators tend to have positive effects on plant growth regardless of whether their prey is a Pest or Mutualist.

\section{Generally positive effects of soil organisms on plant growth}

An important finding of this review was that the net effect of adding soil organisms was positive and similarly that most soil trophic levels increased plant growth. This finding was supported by results from experiments that added multiple trophic levels (i.e. Community treatments): Community treatments increased plant growth. This positive Community effect did not occur because Community treatments included mycorrhizae. Only 11 of the 88 Community experiments included mycorrhizae and removing these 11 experiments did not decrease the positive effects of Community treatments on plant growth (data not shown). Rather, our results suggest that maintaining or increasing soil organism abundance had an intrinsically positive effect on plant growth regardless of the combination of soil organisms added.

Misclassification of organisms is a concern with this type of review. This is a particular concern with soil organisms because, for example, many organisms that were once thought to be Decomposers have been found to derive much of their carbon from microbial biomass and not plant materials (Korobushkin et al. 2014; but see Ngosong et al. 2011). However, we believe our results are robust to misclassification for several reasons. First, most taxonomic groups and trophic categories had positive effects on plant growth. For example, our Decomposer category as defined by the authors from which data was collected was primarily comprised of bacteria, Collembola and Enchytraeids. Collembola and Enchytraeids may have been misclassified as Decomposers but moving data from these categories to the Predator of Mutualist category would not have changed our finding that both Decomposers and Predators of Mutualists increased plant growth. Similarly, Earthworms could have been placed in the Decomposer or Predator of Mutualist categories or a mix of both, but this would not have changed our results. In the Pest pathway, Pests and Predators of Pests tend to be larger and more specialized in their feeding so our finding of trophic cascades in the Pest pathway is less likely to be biased by misclassification than results in the Mutualist pathway. More broadly, most soil organisms demonstrated positive effects on plant growth. As a result, most attempts to re-classify our dataset will produce positive effects of soil organisms on plant growth. Only those organisms identified by authors as herbivores and pathogens demonstrated negative effects on plant growth. This is the major and critical finding from this review and the reason we further reduce our 'trophic categories' to very simple Mutualist and Pest categories (Fig. 5).

There is a growing body of research that uses stable isotope techniques to define the trophic position of soil organisms (Scheu and Falca 2000, Maraun et al. 2011, Korobushkin et al. 2014). Unfortunately, isotope-derived estimates of trophic positions were available for only $15 \%$ of the soil organisms in our dataset. As a larger body of isotope-based studies becomes available it will be possible to revisit the dataset from this study to confirm or redefine the trophic positions of different organisms. Such re-classification of the dataset is likely to change the quantitative estimate of the effects of different trophic categories but will not change the fact that most soil organism additions increased plant growth.

That soil organisms would have a net positive effect on plant growth stands in contrast to a long history of successful crop rotation and conclusions drawn from soil sterilization and plant-soil feedback studies (Troelstra et al. 2001, Kulmatiski et al. 2008, Van der Putten et al. 2013). We suggest several reasons why this past evidence may be misleading. First, pathogen accumulation is more likely in crop systems where soil and plant communities have been dramatically simplified than in more diverse plant-soil systems that are better buffered from pest outbreaks (Bradford et al. 2002, Balvanera et al. 2006, Kulmatiski et al. 2012). Second, positive effects of soil sterilization on plant growth have previously been used to suggest that soil organisms must have a net negative effect on plant growth (Troelstra et al. 2001). This interpretation has been criticized because sterilization causes a flush of nutrients that can also increase plant growth (Troelstra et al. 2001). Further, it is clear that plants could not survive indefinitely without the nutrient recycling performed by decomposers. We believe the current meta-analysis provides a better understanding of the effects of soil organisms and trophic diversity on plant growth than these previous lines of evidence because the current meta-analysis reviewed the effects of explicit soil organism additions and removals.

The broad pattern of soil organism effects was consistent among plant types and ecosystems, but these effects tended to be larger and more positive for Forbs, Trees and Forests than for Grasses and Grasslands. It is not clear why Forbs and Trees realized larger and more positive responses than Grasses. It is possible that long-lived Trees require better defenses or symbioses than shorter-lived species and this is a potential explanation for their more-positive responses than Grasses but does not help explain why Forbs demonstrated similar responses to Trees. It is possible that more nutrient-rich Forbs require greater defense or symbioses than Grasses. Testing and resolving these differences may 
provide a fundamental improvement in understanding of how different plant types interact with soil organisms and other plants.

Human activities have been simplifying ecosystems around the world (Postma-Blaauw et al. 2010). This 'trophic downgrading' typically has the greatest effects on top-level predators (Estes et al. 2011, Wollrab et al. 2012). In aboveground systems, this has been found to alter plant growth and the provision of ecosystem services (Schmitz et al. 2000, Hooper et al. 2005), but it has not been clear how simplifying soil food webs will affect plant growth (Bradford et al. 2002, Hunt and Wall 2002). Our results suggest that simplifying soil trophic diversity is likely to decrease plant growth, though it is critical to note that our dataset lacks results from experiments that added toplevel soil predators. It is also important to note that we reviewed the effects of soil organisms on plant growth. The effects of soil organisms on plant diversity remain poorly resolved (Bever et al. 1997, van der Heijden et al. 1998, Bever 1999, Bagchi et al. 2014).

Results have to be taken with caution. Perhaps most importantly, experiments reviewed here were not necessarily representative of the abundance of different soil organisms in field soils. For example, our review contained only 40 experiments that manipulated plant pathogens but over 500 experiments that manipulated mycorrhizal fungi. Thus, analyses of the full dataset may underestimate the net effect of pathogens and overestimate the effect of plant symbionts. Guild-level analyses were buffered against this bias but our estimate of dataset-wide effects of soil organism addition (i.e. a $29 \%$ increase in plant growth) is biased by the number of studies in different categories. New studies explicitly testing the relative importance of positive and negative effects of soil organisms will be needed but our review as well as results from experiments where multiple trophic categories were added (i.e. Community treatments) both suggest that the net effect of soil organism additions on plant growth is positive.

A second reason for caution in interpreting results is that most experiments were performed with plant monocultures or simple plant communities that are more likely to show responses to herbivory and trophic dynamics than diverse communities that are buffered from these effects (Bradford et al. 2002, Balvanera et al. 2006, Kulmatiski et al. 2012). As with any meta-analysis, there is likely to be a bias in the literature for researchers to study soil organisms that are expected to cause a change in plant growth. These concerns, however, are common among most reviews of the ecological literature. As a result, we suggest it is appropriate to compare our results to those in other similar reviews (Schmitz et al. 2000, Shurin et al. 2002, Sackett et al. 2010) but we recognize that the effects in all of these reviews may be exaggerated relative to effects that would be observed for ecosystem-level responses in diverse plant communities (Bradford et al. 2002, Balvanera et al. 2006).

In summary, our results suggest that 1) trophic cascades do occur in soils and 2) soil organisms tend to have positive effects on plant growth. Results also suggest that soil trophic diversity may be used as a tool for maintaining plant productivity, particularly for Forbs and Trees. Future studies testing 1) soil trophic effects on different plant types, 2) the effects of top-level trophic categories on plant growth, and 3) soil trophic effects on plant coexistence are warranted.

Acknowledgements - Statement of authorship: AK conceived of the research, collected and analyzed data and prepared the manuscript. JS performed statistical analyses and interpretation. Remaining authors collected and reviewed data and helped prepare the manuscript.

Thanks to the Utah Agricultural Experiment Station and USU Ecology Center.

\section{References}

Adams, D. C. et al. 1997. Resampling tests for meta-analysis of ecological data. - Ecology 78: 1277-1283.

Bagchi, R. et al. 2014. Pathogens and insect herbivores drive rainforest plant diversity and composition. - Nature 506: $85-88$.

Balvanera, P. et al. 2006. Quantifying the evidence for biodiversity effects on ecosystem functioning and services. - Ecol. Lett. 9: $1146-1156$

Bancroft, B. A. et al. 2007. Effects of UVB radiation on marine and freshwater organisms: a synthesis through meta-analysis. - Ecol. Lett. 10: 332-345.

Bever, J. D. 1999. Dynamics within mutualism and the maintenance of diversity: inference from a model of interguild frequency dependence. - Ecol. Lett. 2: 52-62.

Bever, J. D. et al. 1997. Incorporating the soil community into plant population dynamics: the utility of the feedback approach. - J. Ecol. 85: 561-573.

Bever, J. D. et al. 2010. Rooting theories of plant community ecology in microbial interactions. - Trends Ecol. Evol. 25: 468-478.

Blossey, B. and Hunt-Joshi, T. R. 2003. Belowground herbivory by insects: influence on plants and aboveground herbivores. - Annu. Rev. Entomol. 48: 521-547.

Borer, E. T. et al. 2005. What determines the strength of a trophic cascade? - Ecology 86: 528-537.

Bradford, M. A. et al. 2002. Impacts of soil faunal community composition on model grassland ecosystems. - Science 298: 615-618.

Chen, Y. et al. 2006. Effect of scleroderma spore density and age on mycorrhiza formation and growth of containerized Eucalyptus globulus and E. urophylla seedlings. - New For. 31: 453-467.

Clarholm, M. 1985. Interactions of bacteria, protozoa and plants leading to mineralization of soil nitrogen. - Soil Biol. Biochem. 17: 181-187.

Denno, R. F. et al. 2008. Potential for entomopathogenic nematodes in biological control: a meta-analytical synthesis and insights from trophic cascade theory. - J. Nematol. 40: 61-72.

DuMouchel, W. H. and Harris, J. E. 1983. Bayes methods for combining the results of cancer studies in humans and other species. - J. Am. Stat. Ass. 78: 293-308.

DuMouchel, W. and Normand, S.-L. 2000. Computer-modeling and graphical strategies for meta-analysis. - In: Stangl, D. and Berry, D. A. (eds), Meta-analysis in medicine and health policy. Chapman and Hall/CRC Biostatistics Series, pp. 127-178.

Egger, M. et al. 1997. Bias in meta-analysis detected by a simple, graphical test. - Brit. Med. J. 315: 629-634.

Eisenhauer, N. et al. 2012. Increasing plant diversity effects on productivity with time due to delayed soil biota effects on plants. - Basic Appl. Ecol. 13: 571-578. 
Estes, J. A. et al. 2011. Trophic downgrading of planet earth. - Science 333: 301-306.

Ettema, C. H. and Wardle, D. A. 2002. Spatial soil ecology. - Trends Ecol. Evol. 17: 177-183.

Fierer, N. et al. 2009. Searching for unifying principles in soil ecology. - Soil Biol. Biochem. 41: 2249-2256.

Franklin, R. B. and Mills, A. L. 2003. Multi-scale variation in spatial heterogeneity for microbial community structure in an eastern Virginia agricultural field. - FEMS Microbiol. Ecol. 44: 335-346.

Gleser, L. J. and Olkin, I. 1994. Stochastically dependent effect sizes. - In: Cooper, H. and Hedges, L. V. (eds), The handbook of research synthesis and meta-analysis. Russell Sage Foundation, pp. 357-376.

Gurevitch, J. and Hedges, L. V. 1999. Statistical issues in ecological meta-analyses. - Ecology 80: 1142-1149.

Gurevitch, J. et al. 2001. Meta-analysis in ecology. - Adv. Ecol. Res. 32: 199-247.

Hairston, N. G. et al. 1960. Community structure, population control, and competition. - Am. Nat. 44: 421-425.

Hedges, L. V. and Olkin, I. 1985. Statistical methods for meta-analysis. - Academic Press.

Hedlund, K. and Öhrn, M. S. 2000. Tritrophic interactions in a soil community enhance decomposition rates. - Oikos 88: 585-591.

Hooper, D. U. et al. 2005. Effects of biodiversity on ecosystem functioning: a consensus of current knowledge. - Ecol. Monogr. 75: 3-35.

Hunt, H. W. and Wall, D. H. 2002. Modelling the effects of loss of soil biodiversity on ecosystem function. - Global Change Biol. 8: 33-50.

Ingham, R. E. et al. 1985. Interactions of bacteria, fungi, and their nematode grazers: effects on nutrient cycling and plant growth. - Ecol. Monogr. 55: 119-140.

Jarosz, A. M. and Davelos, A. L. 1995. Effects of disease in wild plant populations and the evolution of pathogen aggressiveness. - New Phytol. 129: 371-387.

Klarner, B. et al. 2013. Trophic diversity and niche partitioning in a species rich predator guild - natural variations in stable isotope ratios (C-13/C-12, N-15/N-14) of mesostigmatid mites (Acari, Mesostigmata) from central European beech forests. - Soil Biol. Biochem. 57: 327-333.

Klironomos, J. N. 2002. Feedback with soil biota contributes to plant rarity and invasiveness in communities. - Nature 417: 67-70.

Klironomos, J. N. 2003. Variation in plant response to native and exotic arbuscular mycorrhizal fungi. - Ecology 84: 2292-2301.

Klironomos, J. N. and Kendrick, W. B. 1996. Palatability of microfungi to soil arthropods in relation to the functioning of arbuscular mycorrhizae. - Biol. Fertil. Soils 21: 43-52.

Knight, T. M. et al. 2006. Predation on mutualists can reduce the strength of trophic cascades. - Ecol. Lett. 9: 1173-1178.

Korobushkin, D. I. et al. 2014. Isotopic niche $(\delta 13 \mathrm{C}$ and $\delta 15 \mathrm{~N}$ values) of soil macrofauna in temperate forests. - Rapid Comm. Mass Spectrom. 28: 1303-1311.

Kulmatiski, A. et al. 2008. Plant-soil feedbacks: a meta-analytical review. - Ecol. Lett. 11: 980-992.

Kulmatiski, A. et al. 2012. Plant-soil feedbacks provide an additional explanation for diversity-productivity relationships. - Proc. R. Soc. B 279: 3020-3026.

Lang, B. et al. 2014. Effects of environmental warming and drought on size-structured soil food webs. - Oikos 123: 1224-1233.

Lavelle, P. et al. 1997. Soil function in a changing world: the role of invertebrate ecosystem engineers. - Eur. J. Soil Biol. 33: $159-193$.

Lenoir, L. et al. 2007. Bottom-up or top-down control in forest soil microcosms? Effects of soil fauna on fungal biomass and C/N mineralisation. - Biol. Fertil. Soils 43: 281-294.
Letourneau, D. K. et al. 2009. Effects of natural enemy biodiversity on the suppression of arthropod herbivores in terrestrial ecosystems. - Annu. Rev. Ecol. Evol. Syst. 40: 573-592.

Louis, T. A. and Zelterman, D. 1994. Bayesian approaches to research synthesis. - In: Cooper, H. and Hedges, L. V. (eds), The handbook of research synthesis. Russell Sage Foundation, pp. 411-422.

Maraun, M. et al. 2011. Stable isotopes revisited: their use and limits for oribatid mite trophic ecology. - Soil Biol. Biochem. 43: 877-882.

Mikola, J. and Setälä, H. 1998. No evidence of trophic cascades in an experimental microbial-based soil food web. - Ecology 79: 153-164.

Moore, J. C. et al. 2003. Top-down is bottom-up: does predation in the rhizosphere regulate aboveground dynamics? - Ecology 84: 846-857.

Moore, J. C. et al. 2004. Detritus, trophic dynamics and biodiversity. - Ecol. Lett. 7: 584-600.

Morris, W. F. et al. 2007. Direct and interactive effects of enemies and mutualsts on plant performance: a meta-analysis. - Ecology 88: 1021-1029.

Ngosong, C. et al. 2011. Tracking Collembola feeding strategies by the natural C-13 signal of fatty acids in an arable soil with different fertilizer regimes. - Pedobiologia 54: 225-233.

Nieminen, J. K. 2008. Soil animals and ecosystem processes: how much does nutrient cycling explain? - Pedobiologia 51: 367-373.

Paine, R. T. 1980. Food webs: linkage, interaction strength and community infrastructure. - J. Anim. Ecol. 49: 667-685.

Polis, G. A. 1999. Why are parts of the world green? Multiple factors control productivity and the distribution of biomass. - Oikos 86: 3-15.

Polis, G. A. and Strong, D. R. 1996. Food web complexity and community dynamics. - Am. Nat. 147: 813-846.

Postma-Blaauw, M. B. et al. 2010. Soil biota community structure and abundance under agricultural intensification and extensification. - Ecology 91: 460-473.

Preisser, E. L. 2003. Field evidence for a rapidly cascading underground food web. - Ecology 84: 869-874.

Rosenberg, M. S. 2005. The file-drawer problem revisited: a general weighted method for calculating fail-safe numbers in metaanalysis. - Evolution 59: 464-468.

Rosenberg, M. S. et al. 2000. MetaWin: statistical software for meta-analysis. - Sinauer.

Rosenheim, J. A. 1998. Higher-order predators and the regulation of insect herbivore populations. - Annu. Rev. Entomol. 43: 421-447.

Rosenthal, R. 1979. The "file drawer problem" and tolerance for null results. - Psychol. Bull. 86: 638-641.

Sackett, T. E. et al. 2010. Linking soil food web structure to above- and belowground ecosystem processes: a meta-analysis. - Oikos 119: 1984-1992.

Scheu, S. and Falca, M. 2000. The soil food web of two beech forests (Fagus sylvatica) of contrasting humus type: stable isotope analysis of a macro- and a mesofauna-dominated community. - Oecologia 123: 285-296.

Schmitz, O. J. et al. 2000. Trophic cascades in terrestrial systems: a review of the effects of carnivore removals on plants. - Am. Nat. 155: 141-153.

Schmitz, O. J. et al. 2006. Alternative dynamic regimes and trophic control of plant succession. - Ecosystems 9: 659-672.

Schneider, F. D. et al. 2012. Body mass constraints on feeding rates determine the consequences of predator loss. - Ecol. Lett. 15: 436-443.

Shurin, J. B. et al. 2002. A cross-ecosystem comparison of the strength of trophic cascades. - Ecol. Lett. 5: 785-791.

Sin, H. et al. 2008. An invasive frog, Eleutherodactylus coqui, increases new leaf production and leaf litter decomposition rates through nutrient cycling in Hawaii. - Biol. Invas. 10: 335-345. 
Singh, U. B. et al. 2012. Evaluation of biocontrol potential of Arthrobotrys oligospora against Meloidogyne graminicola and Rhizoctonia solani in Rice (Oryza sativa L.). - Biol. Control 60: 262-270.

Stevens, J. R. and Nicholas, G. 2009. metahdep: meta-analysis of hierarchically dependent gene expression studies. - Bioinformatics 25: 2619-2620.

Stevens, J. R. and Taylor, A. M. 2009. Hierarchical dependence in meta-analysis. - J. Educ. Behav. Stat. 34: 46-73.

Strickland, M. S. et al. 2013. Trophic cascade alters ecosystem carbon exchange. - Proc. Natl Acad. Sci. USA 110: 11035-11038.

Strong, D. R. and Frank, K. T. 2010. Human involvement in food webs. - In: Gadgil, A. and Liverman, D. M. (eds), Annual review of environment and resources, Vol. 35, pp. 1-23.

Toepfer, S. et al. 2009. A review of the natural enemies of beetles in the subtribe Diabroticina (Coleoptera: Chrysomelidae): implications for sustainable pest management. - Biocontrol Sci. Technol. 19: 1-65.

Troelstra, S. R. et al. 2001. Interpretation of bioassays in the study of interactions between soil organisms and plants: involvement of nutrient factors. - New Phytol. 150: 697-706.

Tummers, B. 2006. Datathief III. - < http://datathief.org/>.

van der Heijden, M. G. A. et al. 1998. Mycorrhizal fungal diversity determines plant biodiversity, ecosystem variability and productivity. - Nature 396: 69-72.

Supplementary material (available online as Appendix oik.01767 at <www.oikosoffice.org/readers/appendix $>$ ). Appendix 1-2.
Van der Putten, W. H. et al. 2004. Trophic interactions in a changing world. - Basic Appl. Ecol. 5: 487-494.

Van der Putten, W. H. et al. 2013. Plant-soil feedback: the past, the present and future challenges. - J. Ecol. 101: $265-276$.

Van Tol, R. W. H. M. et al. 2001. Plants protect their roots by alerting the enemies of grubs. - Ecol. Lett. 4: 292-294.

Verhoef, H. A. and Brussaard, L. 1990. Decomposition and nitrogen mineralization in natural and agroecosystems: the contribution of soil animals. - Biogeochemistry 11: 175-211.

Wall, D. H. and Moore, J. C. 1999. Interactions underground: soil biodiversity, mutualism and ecosystem processes. - BioScience 49: 109-117.

Wardle, D. A. 2006. The influence of biotic interactions on soil biodiversity. - Ecol. Lett. 9: 870-886.

Wardle, D. A. et al. 1995. The detritus food-web and the diversity of soil fauna as indicators of disturbance regimes in agroecosystems. - Plant Soil 170: 35-43.

Wollrab, S. et al. 2012. Simple rules describe bottom-up and top-down control in food webs with alternative energy pathways. - Ecol. Lett. 15: 935-946.

Zhao, C. et al. 2013. Predatory beetles facilitate plant growth by driving earthworms to lower soil layers. - J. Anim. Ecol. 82: 749-758. 